\title{
O sofrimento psíquico de mulheres vítimas de violência sexual
}

\author{
The psychological suffering of women victims of sexual violence \\ El sufrimiento psicológico de las mujeres víctimas de violencia sexual
}

Recebido: 25/06/2021 | Revisado: 17/07/2021 | Aceito: 24/07/2021 | Publicado: 01/08/2021

Maria Eduarda Wanderley de Barros Silva

ORCID: https://orcid.org/0000-0002-4642-3282

Universidade Federal de Campina Grande, Brasil

E-mail: eduarda.wanderley@outlook.com

Vitoria Vilas Boas da Silva Bomfim

ORCID: https://orcid.org/0000-0003-4897-0279

Centro Universitário Jorge Amado, Brasil

E-mail: pesquisaclinica9@gmail.com

Thaís Andrade dos Santos

ORCID: https://orcid.org/0000-0002-9669-7468

Universidade Uninassau, Brasil

E-mail: thaisandrade24@icloud.com

Tainá Oliveira de Araújo

ORCID: https://orcid.org/0000-0002-5069-7243

Universidade Federal de Campina Grande, Brasil

E-mail: tainaaraujo@gmail.com

Wendel Vinícius Lourenço Rodrigues

ORCID: https://orcid.org/0000-0002-0103-9180

Universidade Federal de Campina Grande, Brasil

E-mail: del6_5@hotmail.com

Daniela de Lira Silva

ORCID: https://orcid.org/0000-0001-8145-2508

Universidade Federal de Pernambuco, Brasil

E-mail: daniela.lirasilva@ufpe.br

Yorrane Kelly Gomes Alves

ORCID: https://orcid.org/0000-0003-3835-0885

Universidade Federal de Campina Grande, Brasil

E-mail: yorranekelly11@gmail.com

Yasmin Santana do Nascimento

ORCID: https://orcid.org/0000-0001-7828-1285

Universidade Maurício de Nassau, Brasil

E-mail: yasminnascimento@hotmail.com

Jean Scheievany da Silva

ORCID: https://orcid.org/0000-0002-3693-3676

Universidade Federal de Pernambuco, Brasil

E-mail: jean.alves@ufpe.br

Fernanda Félix de Araújo Varela

ORCID: https://orcid.org/0000-0001-6550-9113

Universidade Federal de Campina Grande, Brasil

E-mail: fernandafeliix@hotmail.com

Suellen Stephanie Azevedo

ORCID: https://orcid.org/0000-0002-6758-0703

Universidade Federal de Pernambuco, Brasil

E-mail: suellenbbb.sb@gmail.com

Maria Gabryelle da Silva Soares

ORCID: https://orcid.org/0000-0003-2575-8267

Universidade Federal de Campina Grande, Brasil

E-mail: gabryelle8685@gmail.com

\section{Resumo}

Pautada na violência de gênero, a violência contra a mulher constitui uma das principais formas de violação dos direitos humanos, o que abrange o direito à vida, integridade física e saúde. Objetivou identificar os impactos que causam sofrimento psíquico nas mulheres vítimas de violência sexual. O método utilizado foi a revisão integrativa da literatura. Para pesquisar os artigos a serem revisados, foram utilizadas as bases de dados PubMed, Biblioteca Virtual de Saúde e Scientific Electronic Library Online. Realizado no primeiro semestre de 2021 entre os meses de maio e junho, com os Descritores em Ciências da Saúde: "Mulheres"; "Saúde Mental"; e "Violência contra a Mulher". (o que tem grandes consequências? Arrumar essa frase) Tem grandes consequências para a saúde, não apenas para as mulheres e crianças, mas também para suas famílias, além de contribuir para o fardo global de problemas de saúde, 
aumentando as taxas de morbidade e mortalidade das mulheres, incluindo trauma psicológico, medo, depressão, suicídio, assassinato, dor crônica, lesões, fraturas, deficiência, gravidez indesejada, uso inadequado de anticoncepcionais, além do risco de adquirir HIV e outras infecções sexualmente transmissíveis. Diante da condição de estresse psicológico e fragilidade pós trauma, o sofrimento psíquico das mulheres abusadas sexualmente torna-se temática significante dentro da assistência em saúde através da multidisciplinaridade, por se tratar do bem-estar biopsicossocial das vítimas, evidenciando um olhar mais direcionado para a integridade dos direitos sociais e de saúde de cada mulher objetivando diminuir o sofrimento psicológico vivenciado comumente entre as mulheres violentadas.

Palavras-chave: Mulheres; Violência contra a mulher; Saúde mental.

\begin{abstract}
Based on gender violence, violence against women is one of the main forms of violation of human rights, which includes the right to life, physical integrity and health. It aimed to identify the impacts that cause psychological distress in women victims of sexual violence. The method used was an integrative literature review. To search the articles to be reviewed, the PubMed, Virtual Health Library and Scientific Electronic Library Online databases were used. Carried out in the first half of 2021 between May and June, with the Health Sciences Descriptors: "Women"; "Mental health"; and "Violence against Women". (What has big consequences? Fix this sentence) It has big health consequences, not only for women and children, but also for their families, in addition to contributing to the global burden of health problems, increasing morbidity and rates. women's mortality, including psychological trauma, fear, depression, suicide, murder, chronic pain, injuries, fractures, disability, unwanted pregnancy, inappropriate use of contraceptives, and the risk of acquiring HIV and other sexually transmitted infections. Faced with the condition of psychological stress and post-trauma frailty, the psychological suffering of sexually abused women becomes a significant theme within health care through multidisciplinarity, as it deals with the biopsychosocial well-being of the victims, evidencing a more focused look at the integrity of the social and health rights of each woman, aiming to reduce the psychological suffering commonly experienced among abused women.
\end{abstract}

Keywords: Women; Violence against women; Mental health.

\title{
Resumen
}

Con base en la violencia de género, la violencia contra la mujer es una de las principales formas de violación de los derechos humanos, que incluye el derecho a la vida, la integridad física y la salud. Tuvo como objetivo identificar los impactos que provocan malestar psicológico en mujeres víctimas de violencia sexual. El método utilizado fue una revisión integradora de la literatura. Para la búsqueda de los artículos a revisar se utilizaron las bases de datos PubMed, Virtual Health Library y Scientific Electronic Library Online. Realizado en el primer semestre de 2021 entre mayo y junio, con los Descriptores de Ciencias de la Salud: "Mujeres"; "Salud mental"; y "Violencia contra la mujer". (¿Qué tiene grandes consecuencias? Arregle esta sentencia) Tiene grandes consecuencias para la salud, no solo para las mujeres y los niños, sino también para sus familias, además de contribuir a la carga global de problemas de salud, aumentando la morbilidad y las tasas. trauma psicológico, miedo, depresión, suicidio, asesinato, dolor crónico, lesiones, fracturas, discapacidad, embarazo no deseado, uso inadecuado de anticonceptivos, además del riesgo de contraer el VIH y otras infecciones de transmisión sexual. Frente a la condición de estrés psicológico y fragilidad postraumática, el sufrimiento psicológico de las mujeres abusadas sexualmente se convierte en un tema significativo dentro de la atención de la salud a través de la multidisciplinariedad, ya que se trata del bienestar biopsicosocial de las víctimas, evidenciando una mirada más focalizada en el integridad de los derechos sociales y de salud de cada mujer, con el objetivo de reducir el sufrimiento psicológico comúnmente experimentado entre las mujeres maltratadas.

Palabras clave: Mujeres; La violencia contra las mujeres; Salud mental.

\section{Introdução}

A violência em todos os seus tipos e abordagens se configura como um fenômeno que atinge as mulheres de diversas classes sociais, estado civil, escolaridade, idade, raça, origem e orientação sexual. Dessa forma, entre os diferentes tipos de violência, ressalta-se a violência sexual compreendida como uma ação que obriga a mulher manter relações sexuais sem seu devido consentimento utilizando a força e anulando assim sua vontade (Trigueiro, Silva, Merighi, Oliveira \& Jesus, 2017).

Pautado na violência de gênero, a violência contra a mulher constitui uma das principais formas de violação dos direitos humanos, o que abrange o direito à vida, integridade física e saúde. Assim, é perceptível que nas diversas apresentações da violência em graus e formas, a integridade bem como o bem-estar dessas mulheres violentadas são afetados causando impactos e distintas consequências, tanto física quanto psicológica (Nelon, Pedro, Gilreath, Patterson, Holden, \& Esquivel, 2019). 
O alto índice de violência sexual contra as mulheres é um problema de saúde pública preocupante, pois o ataque sexual é definido como uma violência forçada ou coagida, contato sexual comportamento sem consentimento violando assim a integridade sexual expondo a uma variedade de resultados negativos para a saúde. Segunda causa de morte no obituário geral, primeira nas faixas etárias dos 5 aos 39 anos, pode causar lesões e traumas físicos e emocionais, deixando um lastro de problemas, alguns diagnosticáveis, outros difusos, todos de elevada magnitude, afetando indivíduos, famílias, grupos e a sociedade como um todo (Mcqueen, Oikonen, Miller \& Chambers, 2021; Oliveira, Jorge \& Salete, 2007).

Dados do último relatório do Instituto de Pesquisa Econômica Aplicada (IPEA) sobre o Atlas da Violência, que traz informações sobre o número de feminicídios, mostram que só em 2016, 4.645 mulheres foram assassinadas no Brasil, correspondendo a 4,5 mortes por 100.000 mulheres. E, dados de 2017 indicam o número de feminicídios em termos absolutos no Brasil, com 1.133 vítimas confirmadas em 2017. Isso mostra que a violência atinge picos elevados de preocupação social e que sua repercussão na vida e na intimidade de muitas mulheres acabam interferindo negativamente nas suas relações interpessoais, psicológicas e sociais (Taís, Lúcia, Silva \& Carla 2020).

Contudo, o abuso físico repercute também na sexualidade feminina. Sierra sugere que o abuso não físico pode ter a capacidade de prever o funcionamento sexual das mulheres. Porém, a experiência de abuso físico implica uma piora nas dimensões do funcionamento sexual nas mulheres. Ter sofrido abuso na relação com o parceiro tem sido relacionado às dimensões do funcionamento sexual, sem falar que o quadro de violência física pode ser um preditor negativo do desejo sexual proporcionando negações e baixa autoestima por parte da mulher (Juan, Ana I, Ana \& Oscar 2021).

Com isso a violência sexual se apresenta de diversas formas: prática do ato sexual indesejável, tráfico sexual, exploração sexual e aliciamento, fazendo com que as mulheres apresentam mudanças no comportamento com a baixa autoestima, depressão, isolamento e constrangimento para dialogar sobre o ocorrido. De acordo com as informações da atenção de integração à saúde, o atendimento a essas mulheres violentadas deve estar embasado em conhecimentos científicos, epidemiológicos e tecnológicos para que possam ser respeitadas suas peculiaridades, de acordo com cada caso e de forma legalizada (Neta, Guimarães, Farias \& Santos, 2020).

A violência sexual por parceiro íntimo é um produto das relações de gênero, estruturas e práticas de poder masculino. A coerção sexual e a violência são prevalentes em muitos casamentos, e a maioria das mulheres suporta o abuso dos parceiros silenciosamente devido a normas sociais e culturais restritivas e estigma associado à separação ou divórcio. O sexo não consensual no casamento faz parte da paisagem cultural mais ampla de muitas sociedades onde se acredita que o sexo no casamento é um direito do homem sendo essa uma visão direta do patriarcado, instigando ainda mais submissão sexual feminina frente a um costume cultural que pode ser encontrado no mundo todo (Zaina, Gerry, Diana, Esther, Donati, Annapoorna, Saidi \& Heidi 2020).

Devido à magnitude que o fenômeno atinge, este contribui para o surgimento de agravantes na saúde da mulher apresentando sintomas de dimensões psicológicas, comportamentais, depressão e suicídio afetando, assim, o sofrimento psíquico da mulher violentada sexualmente. Assim, é preciso que os profissionais de saúde estejam preparados para os impactos e consequências que a violência sexual acarreta para a saúde psíquica das mulheres violentadas (Nelon, Pedro, Gilreath, Patterson, Holden, \& Esquivel, 2019; Oram, Khalifeh, \& Howard, 2017).

A violência sexual, independentemente de ser com pessoa conhecida ou não, quando ela não é consentida altera o cotidiano dessas mulheres trazendo diversas consequências para sua saúde física, reprodutiva, social e psicológica sobretudo entre as mais jovens. Assim, o sofrimento psíquico e mal-estar psicológico se refere a percepção de pensamentos e sentimentos que refletem à carga de estresse, desânimo, medo, baixa autoestima, condições que demonstram o sofrimento psíquico e emocional produzido pelas próprias tensões e contradições entre as experiências vividas. 
Justifica-se a realização deste estudo por ser uma problemática social e de segurança, de grande relevância, atual e de extremo interesse para a saúde pública, devido ao alto índice de casos, mortalidade e consequências na saúde dos indivíduos e nas relações sociais, expressa nas lesões físicas e psicológicas decorrentes. Torna-se uma demanda expressiva e decorrente nos serviços de saúde, vista a intensificação e gravidade das violências, trazendo altos custos financeiros e humanos. Diante do exposto, este estudo teve como objetivo identificar os impactos que causam sofrimento psíquico nas mulheres vítimas de violência sexual.

\section{Metodologia}

O método utilizado foi a revisão integrativa da literatura. A questão norteadora foi: "Quais são os sofrimentos psíquicos relatados pelas mulheres vítimas de violência sexual?".

Se entende por revisão integrativa da literatura um levantamento sobre as principais teorias que norteiam o trabalho científico, buscando solução para o problema analisando, produzindo ou explicando o objeto a ser investigado (Ercole, Melo, \& Alcoforado, 2014).

Assim, os critérios para inclusão dos estudos selecionados foram: artigos disponibilizados na íntegra e gratuitamente, nos idiomas inglês e português publicados no período entre 2016 e 2021 e que abordassem sobre a temática proposta. Foram excluídos os artigos cujo texto completo não estivesse disponível na modalidade gratuita, estudos secundários, carta ao leitor, teses e dissertações. Utilizou-se as seguintes combinações de descritores: "Mulheres", "Saúde Mental" e "Violência contra a Mulher" sendo separados pelo operador "AND", garantindo a inclusão de todos os artigos que fossem referentes ao tema proposto.

A pesquisa foi realizada de forma independente, por meio do cruzamento nas bases selecionadas. Desta forma, foram encontrados 1038 artigos indexados nas bases de dados consultadas, sendo: 88 na base SciELO a partir do cruzamento dos DECS (Descritores em Ciência da Saúde) citados anteriormente, 247 na base BVS e 703 na PubMed. Após filtragem, análise criteriosa dos artigos e critérios de exclusão, que se adequaram a questão norteadora e são objeto desta pesquisa de revisão integrativa, permaneceram, na amostra final, 9 artigos sendo 4 da SciELO, 4 da PubMed e 2 da BVS.

O Quadro 1 está representado pela seleção dos artigos pesquisados, excluídos e selecionados por bases de dados.

Quadro 1. Distribuição dos artigos selecionados para revisão, segundo base de dados.

\begin{tabular}{|c|c|c|c|}
\hline \multirow{2}{*}{ ARTIGOS } & \multicolumn{3}{|c|}{ BASE DE DADOS } \\
\cline { 2 - 4 } & SCIELO & BVS & PUBMED \\
\hline Pesquisados & 88 & 247 & 703 \\
\hline Excluídos & 84 & 246 & 699 \\
\hline Selecionados & 4 & 2 & 4 \\
\hline Total & \multicolumn{3}{|c}{} \\
\hline
\end{tabular}

Fonte: Autores (2021).

Assim, os artigos foram compilados, sintetizados e organizados de maneira a terem suas principais informações expostas, agrupando-as de maneira sistematizada por meio do programa Microsoft Office Word.

\section{Resultados e Discussão}

A amostragem contou com 10 artigos analisados, que respondiam aos critérios de inclusão previamente estabelecidos. Sendo que estas publicações apresentaram as respostas mais precisas para o objetivo da pesquisa, que estão descritas no quadro 2, na sequência numeração, autores/ano, título e objetivos. 
Quadro 2. Distribuição dos artigos selecionados apresentado autor, título, objetivo, ano. Recife (PE), 2021.

\begin{tabular}{|c|c|c|c|}
\hline No. & Autor/Ano & Título & Objetivo \\
\hline 01 & $\begin{array}{l}\text { Trigueiro, Silva, } \\
\text { Merighi, Oliveira \& } \\
\text { Jesus (2017) }\end{array}$ & $\begin{array}{l}\text { O sofrimento psíquico no cotidiano } \\
\text { de mulheres que vivenciaram a } \\
\text { violência sexual: estudo } \\
\text { fenomenológico. }\end{array}$ & $\begin{array}{l}\text { Compreender as ações do cotidiano de } \\
\text { mulheres que vivenciaram violência sexual. }\end{array}$ \\
\hline 02 & $\begin{array}{l}\text { Nelon, Pedro, Giltreath. } \\
\text { Patterson, Holden \& } \\
\text { Esquivel (2019) }\end{array}$ & $\begin{array}{l}\text { A latent class analysis of the co- } \\
\text { occurrence of sexual violence, } \\
\text { substance use, and mental health in } \\
\text { youth. }\end{array}$ & $\begin{array}{c}\text { O objetivo deste estudo foi compreender a } \\
\text { violência sexual, uso de substâncias e saúde } \\
\text { mental em jovens e verificar se há diferenças } \\
\text { nas experiências entre identidades sexuais, } \\
\text { raças e gêneros. }\end{array}$ \\
\hline 03 & $\begin{array}{l}\text { Oram, Khalifeh \& } \\
\text { Howard (2017) }\end{array}$ & $\begin{array}{l}\text { Violence against women and } \\
\text { mental health. }\end{array}$ & $\begin{array}{l}\text { Nosso objetivo era revisar evidências sobre a } \\
\text { associação entre violência contra mulheres e } \\
\text { saúde mental, a identificação de e resposta à } \\
\text { violência contra a mulher pela saúde mental } \\
\text { serviços e intervenções de saúde mental para } \\
\text { as vítimas e perpetradores de violência contra } \\
\text { as mulheres. }\end{array}$ \\
\hline 04 & $\begin{array}{c}\text { Sharma, Vatsa, } \\
\text { Kalaivani \& Bhardwaj } \\
\text { (2019) }\end{array}$ & $\begin{array}{l}\text { Effects on mental health of } \\
\text { domestic violence against women } \\
\text { in Delhi: A community based study. }\end{array}$ & $\begin{array}{l}\text { Teve como objetivo examinar a associação de } \\
\text { Violência doméstica (DV) com o estado de } \\
\text { saúde mental das mulheres. }\end{array}$ \\
\hline 05 & $\begin{array}{l}\text { Sigurdardottir \& } \\
\text { Halldorsdottir (2021) }\end{array}$ & $\begin{array}{l}\text { Persistent relief: the serious } \\
\text { consequences of sexual violence } \\
\text { against women and men, its search } \\
\text { for inner healing and the meaning } \\
\text { of the \#MeToo movement. }\end{array}$ & $\begin{array}{l}\text { O objetivo de explorar e melhor compreender } \\
\text { a consequências da violência sexual para as } \\
\text { mulheres e sua busca pela cura interior. }\end{array}$ \\
\hline 06 & $\begin{array}{l}\text { Baigorria Judizeli, } \\
\text { Warmling Deise, } \\
\text { Magno Neves, Delziovo } \\
\text { Carmem \& Salema } \\
\text { Coelho (2017) }\end{array}$ & $\begin{array}{l}\text { Prevalence and associated fathers of } \\
\text { sexual violence against women: } \\
\text { systematic review. }\end{array}$ & $\begin{array}{l}\text { Identificar na literatura a prevalência e os } \\
\text { fatores associados à Violência Sexual (VS) } \\
\text { contra adolescentes e mulheres adultas. }\end{array}$ \\
\hline 07 & $\begin{array}{l}\text { Garcia \& Trajano } \\
\text { (2017) }\end{array}$ & $\begin{array}{l}\text { Neurobiology of the media and } \\
\text { specific phobias. }\end{array}$ & $\begin{array}{l}\text { Objetivo de revisar A base neurobiológica } \\
\text { das reações de medo normais e patológicas }\end{array}$ \\
\hline 08 & $\begin{array}{l}\text { Delziovo, Bolsoni, } \\
\text { Nazário \& Coelho } \\
\quad(2017)\end{array}$ & $\begin{array}{c}\text { Características dos casos de } \\
\text { violência sexual contra mulheres } \\
\text { adolescentes e adultas notificados } \\
\text { pelos serviços públicos de saúde em } \\
\text { Santa Catarina, Brasil }\end{array}$ & $\begin{array}{l}\text { Descrever as características dos casos de } \\
\text { violência sexual perpetrada contra mulheres, } \\
\text { notificados por profissionais de saúde nos } \\
\text { anos de } 2008 \text { a 2013, em Santa Catarina. }\end{array}$ \\
\hline 09 & $\begin{array}{l}\text { Pedrosa \& Zanello } \\
\text { (2016) }\end{array}$ & $\begin{array}{l}\text { (In)visibilidade da violência contra } \\
\text { as mulheres na saúde mental }\end{array}$ & $\begin{array}{l}\text { O objetivo deste estudo foi realizar um } \\
\text { levantamento acerca da percepção, crenças e } \\
\text { conhecimentos sobre violência contra as } \\
\text { mulheres e políticas públicas em profissionais } \\
\text { de saúde mental. }\end{array}$ \\
\hline 10 & $\begin{array}{l}\text { Garcia \& Trajano } \\
\text { (2017) }\end{array}$ & $\begin{array}{l}\text { Violência sexual contra mulheres e } \\
\text { saúde mental: um diálogo sobre } \\
\text { norma técnica de prevenção e } \\
\text { tratamento dos agravos resultantes } \\
\text { da violência sexual contra mulheres } \\
\text { e adolescentes }\end{array}$ & $\begin{array}{l}\text { Analisar a Norma Técnica de Prevenção e } \\
\text { Tratamento dos Agravos Resultantes da } \\
\text { Violência Sexual contra Mulheres e } \\
\text { Adolescentes e discorrer a respeito de que } \\
\text { forma ela preserva a saúde mental das vítimas } \\
\text { de violência sexual. }\end{array}$ \\
\hline
\end{tabular}

Fonte: Autores (2021).

Evidenciam-se quatro artigos que no seu título apresentam o nome "saúde mental", quatro com o descritor "mulheres" e apenas dois artigos que no seu título evidencia o descritor “violência contra a mulher". Em seguida, o Quadro 3 apresenta a distribuição dos artigos selecionados segundo o autor, o ano, o idioma, o método do estudo e a base de dados selecionadas. 
Quadro 3. Distribuição dos artigos selecionados apresentado autor/ ano, idioma, método e base de dados. Recife (PE), 2021.

\begin{tabular}{|c|c|c|c|c|}
\hline No. & Autor/ano & Idioma & Método & Base de dados \\
\hline 01 & $\begin{array}{c}\text { Trigueiro, Silva, Merighi, Oliveira \& } \\
\text { Jesus (2017) }\end{array}$ & Português & Pesquisa qualitativa & BVS \\
\hline 02 & $\begin{array}{l}\text { Nelon, Pedro, Giltreath. Patterson, } \\
\text { Holden \& Esquivel (2019) }\end{array}$ & Inglês & Pesquisa qualitativa & PubMed \\
\hline 03 & Oram, Khalifeh \& Howard (2017) & Inglês & Pesquisa qualitativa & PubMed \\
\hline 04 & $\begin{array}{c}\text { Sharma, Vatsa, Kalaivani \& } \\
\text { Bhardwaj (2019) }\end{array}$ & Inglês & $\begin{array}{l}\text { Estudo transversal de caráter } \\
\text { quali-quantitativo }\end{array}$ & SciELO \\
\hline 05 & $\begin{array}{l}\text { Sigurdardottir \& Halldorsdottir } \\
\qquad(2021)\end{array}$ & Inglês & Pesquisa bibliográfica & PubMed \\
\hline 06 & $\begin{array}{c}\text { Baigorria Judizeli, Warmling Deise, } \\
\text { Magno Neves, Delziovo Carmem \& } \\
\text { Salema Coelho (2017) }\end{array}$ & Inglês & Pesquisa bibliográfica & SciELO \\
\hline 07 & Garcia \& Trajano (2017) & Inglês & Estudo de revisão integrativa & PubMed \\
\hline 08 & $\begin{array}{l}\text { Delziovo, Bolsoni, Nazário \& } \\
\text { Coelho (2017) }\end{array}$ & Português & $\begin{array}{c}\text { Caráter descritivo e de série } \\
\text { temporal }\end{array}$ & SciELO \\
\hline 09 & Pedrosa \& Zanello (2016) & Português & Análise de conteúdo & SciELO \\
\hline 10 & Garcia \& Trajano (2017) & Português & Análise de conteúdo & BVS \\
\hline
\end{tabular}

Fonte: Autores (2021).

Identificam-se que cinco artigos foram desenvolvidos em 2017, dois em 2019 e os demais com uma publicação nos anos 2016 e 2021. Desses 7 eram no idioma em português e 3 em inglês. Em relação ao método tem-se 1 estudo transversal, 8 estudos descritivos e 1 revisão sistemática. As bases de dados mais prevalentes foram a PubMed, seguida da SciElo e, por fim, a BVS.

Apesar da violência ser uma categoria vazia e de constante expansão, perpassa as múltiplas categorias de opressão e pode ser entendida como o uso intencional da força física ou do poder, real ou como ameaça, contra outra pessoa ou comunidade, que se configura como um fenômeno biopsicossocial, com repercussões sobre as esferas física, comportamental e cognitiva. Possui especificidade histórica, abrangendo problemas da política, economia, do direito, da moral, das relações humanas e institucional, constituindo-se em uma contraposição à tolerância, ao reconhecimento e à civilização, podendo se manifestar de diversas formas e nos diferentes contextos (Garcia, \& Trajano, 2018).

Partindo desse pressuposto, a violência, em todas as suas formas, caracteriza-se como um fenômeno que atinge mulheres de diferentes classes sociais, origem, idade, estado civil, raça e orientação sexual (Trigueiro, Silva, Merighi, Oliveira \& Jesus, 2017).

Trata-se de um grave problema de saúde pública e uma das principais maneiras de violação aos direitos humanos, o que abrange o direito à vida, à saúde e à integridade física, podendo ser apresentada de diversas formas e graus. Dentre elas destacam-se a violência de gênero, a violência intrafamiliar, a violência doméstica, a violência física, a violência econômica, a violência institucional, a violência psicológica e a violência sexual, que resulta ou pode resultar em dano físico, sexual, psicológico, morte, privação ou sofrimentos as mulheres (Oram, Khalifeh, \& Howard, 2017).

Entre os diferentes tipos de violência, a violência sexual. Segundo a Organização da Saúde (OMS), define-se como violência sexual o ato, tentativa ou investidas sexuais sem consentimento, com o uso ou não de cerceamento, podendo ser 
praticada segundo o organismo, por qualquer pessoa, independentemente da relação com a vítima, e em qualquer cenário, incluindo a casa e o trabalho. Inclui atos de penetração forçada e assédios sexuais como, atos e investidas, na forma opressiva e com pagamento ou favorecimento sexual nas relações hierárquicas. Nesse sentido, este tipo de violência configura-se como uma das mais graves manifestações da desigualdade de gênero, uma vez que, atinge uma quantidade significante de meninas e mulheres globalmente (Baigorria, Warmling, Neves, Delziovo, \& Coelho, 2017).

Segundo dados da Organização Mundial da Saúde (OMS), estima-se que, mundialmente, cerca de 35\% das mulheres sofreram algum tipo de violência física e/ou sexual, chegando a quase $70 \%$ dependendo do país. Além disso, estima-se que três em cada cinco mulheres foram ou serão vítimas de violência, tornando-se uma grande epidemia e uma problemática global, a qual deve ser buscado mecanismos de enfrentamento (Pedrosa, \& Zanello, 2016).

No Brasil, cerca de 15.508 casos de violências contra mulheres foram notificados em 2017, em que 2.010 desses casos foram do tipo violência do tipo sexual, correspondendo aproximadamente $12 \%$ dos casos, onde 950 das notificações foram de adolescentes na faixa etária dos 10 aos 14 anos, configurando cerca de 47\%, já as jovens de 15 aos 19 totalizaram 22\% com 450 dos casos, e as mulheres adultas com 20 anos ou mais representaram aproximadamente 30\% com 610 notificações. Além disso, as mulheres adultas relataram que as agressões ocorreram em suas residências, em via pública, à noite ou pela madrugada, sendo realizada por um único agressor, onde mais de 50\% dos casos teve penetração vaginal resultando em um número maior de lesões físicas e tentativas de suicídio (Delziovo, Bolsoni, Nazário, Coelho, \& Elziovo, 2017).

A violência sexual contra a mulher é um fenômeno múltiplo e complexo que ocorre em uma variedade de situações, como estupro; expressão máxima de violência sexual; compreendido, como ação que obriga a mulher a manter relações sexuais pelo uso da força, anulando a sua vontade. Tem grandes consequências para a saúde, não apenas para as mulheres e crianças, mas também para suas famílias, além de contribuir para o fardo global de problemas de saúde, aumentando as taxas de morbidade e mortalidade das mulheres, incluindo trauma psicológico, medo, depressão, suicídio, assassinato, dor crônica, lesões, fraturas, deficiência, gravidez indesejada, uso inadequado de anticoncepcionais, além do risco de adquirir HIV e outras infecções sexualmente transmissíveis (Sharma, Vatsa, Kalaivani, \& Bhardwaj, 2019).

O medo é algo intrínseco ao ser humano e compõe o seu constitutivo emocional, é caracterizado como um mecanismo de defesa animal fundamental para a sobrevivência que envolve vários processos biológicos para a resposta a eventos ameaçadores (Garcia, 2017). Entretanto, quando esse sentimento negativo se torna crônico e intenso por diversos motivos, como é o caso da violência sexual contra a mulher passará a ser um fator contributivo no desenvolvimento de vários distúrbios psiquiátricos aumentando os níveis de ansiedade e estresse.

Experiências de violência sexual afetam negativamente o bem-estar físico e psicológico de uma pessoa a longo prazo, bem como o desenvolvimento do medo do sexo, perda do interesse sexual e indiferença a assuntos relacionados a atividades sexuais. Em um evento traumático, o corpo responde às principais ameaças, como é o caso da violência sexual, secretando catecolaminas, norepinefrina e dopamina. Este processo biológico é chamado de "resposta de luta ou fuga", sendo sua função primordial a de ativar o organismo e de prepará-lo para o conflito. Entretanto, é mais apropriado chamá-lo de resposta de "luta, fuga ou congelamento", porque a mulher violentada sexualmente possui maior probabilidade de "congelar" e pode levar muito tempo para ela "descongelar" novamente (Sigurdardottir, \& Halldorsdottir, 2021).

O eixo hipotálamo-hipófise-adrenal (HPA) responde com reações envolvendo inúmeros eventos: o hipotálamo libera o hormônio liberador de corticotrofina (CRH), que faz com que a hipófise libere o hormônio adrenocorticotrópico (ACTH), que faz com que as glândulas adrenais liberem corticosteróides. Um evento traumático, como a violência sexual, pode causar estresse de longo prazo, que pode ativar o HPA e o sistema nervoso autônomo. Tal fato, pode manifestar-se como níveis aumentados ou anormalmente baixos de cortisol no sangue e níveis baixos ou elevados de norepinefrina (Sigurdardottir, \& Halldorsdottir, 2021). 
O estresse psicológico pode desencadear a ativação da amígdala e, consequentemente o sistema nervoso autônomo, que desencadeia o sistema imunológico e leva a uma resposta inflamatória. A inflamação pode aumentar o risco de psicopatologia, alterando o metabolismo dos neurotransmissores. Assim, o estresse físico e psicológico decorrente da violência sexual pode causar reações inflamatórias e, portanto, proporcionar o surgimento de novas doenças ou até mesmo agravar aquelas pré-existentes (Sigurdardottir, \& Halldorsdottir, 2021).

A depressão é considerada uma resposta psicológica ao estresse opressor, e inúmeras vezes se sobrepõe a inúmeras patologias, incluindo asma, artrite reumatoide, doenças cardiovasculares, câncer e distúrbios neurológicos (Sigurdardottir, \& Halldorsdottir, 2021).

A saúde tem muitas dimensões, incluindo física, mental, emocional e social. A violência sexual é incrivelmente chocante e tem um impacto negativo significativo em todas as dimensões da saúde da mulher, principalmente no que se refere a esfera mental e emocional (Sigurdardottir, \& Halldorsdottir, 2021).

Uma mulher ou menina que é abusada sexualmente, seja na infância, adolescência ou idade adulta, sofre consequências físicas, mentais e sociais de longo prazo que podem deixar marcas permanente nelas, ocasionando um intenso sofrimento psíquico. Os resultados mais importantes são a dor emocional quase insuportável que ela geralmente esconde dentro de si mesma, depressão, ansiedade, transtorno do estresse pós-traumático, medo, autoconfiança quebrada, autoestima diminuída, vergonha, culpa, comportamento de autolesão, abuso de álcool e drogas, ideação suicida, raiva, tristeza, melancolia, decepção, transtorno de personalidade, transtorno de trauma, memórias difíceis, pesadelos, solidão e isolamento, além de dificuldades de se relacionar amorosamente e de se socializar, o que pode impactar inclusive na vida econômica dessas mulheres (Sigurdardottir, \& Halldorsdottir, 2021).

Desse modo, é notório que a violência sexual é uma contribuinte chave para as disparidades de saúde mental gerando sofrimentos que diminuem o potencial de ação dos sujeitos que estão expostos a eles, além da pertinência dessa temática atualmente, sendo de suma importância o papel desempenhado pelos profissionais de saúde às mulheres vitimizadas.

Nesse contexto, os profissionais de saúde devem estar aptos a ouvir, acolher o sofrimento das mulheres, olhando para além da sintomatologia apresentada pela paciente, promovendo uma atenção integral e contínua à saúde da mulher. Para isso é necessário que eles compreendam os aspectos biológicos, psicológicos e sociais da saúde do sujeito, do ponto de vista de seu contexto social, cultural, familiar e territorial no qual a paciente está inserida (Garcia, \& Trajano, 2018).

Contudo, é preciso estar alerta ao estado psicológico da mulher vítima da violência sexual, pois essa agressão é um evento traumático e se configura como violência física e psicológica de modo simultâneo, com efeitos potencialmente devastadores sobre a saúde física e mental das mulheres, causando danos profundos a sua qualidade de vida e no bem-estar físico, sexual, reprodutivo, emocional, mental e social das vítimas. De modo que, o impacto negativo da violência sexual é refletido em dados à homeostasia da saúde mental imediatos e a longo prazo, e são agravados pela sobreposição de violências sofridas (Baigorria, Warmling, Neves, Delziovo, \& Coelho, 2017).

\section{Considerações Finais}

Diante das situações de violência que as mulheres sofrem comumente dentro da sociedade, é de grande relevância social e de saúde pública que os impactos gerados na vida das vítimas tenham uma atenção mais direcionada a resolubilidade da problemática principal dos abusos, sejam físicos, psicológicos, sociais e sexuais. Os vários tipos de violência contra a mulher atingem diretamente a integridade humana prejudicando sua qualidade de vida e suas relações interpessoais, sendo estes problemas, duradouros e, às vezes, irreparáveis. 
A condição de estresse psicológico e fragilidade pós trauma é uma realidade assustadora de várias mulheres que sentem os diversos impactos gerados nas suas atividades cotidianas, reflexo dos crescentes casos de violências de maneira camuflada dentro do ambiente familiar, social e de trabalho.

O medo e a insegurança das vítimas de violência sexual são alimentados por situações de vulnerabilidade fortemente enraizadas por um sistema de patriarcado incansável na sustentação da superioridade de gênero. Nesse sentido, o sofrimento psíquico das mulheres abusadas sexualmente se torna temática significante dentro da assistência em saúde através da multidisciplinaridade, por se tratar de alterações no bem-estar biopsicossocial da vítima, que inviabiliza novas relações de diversos graus de ligações, sendo evidenciada a necessidade de um olhar mais atencioso para garantia dos direitos humanos integralizados no contexto social e de saúde de cada mulher.

\section{Referências}

Baigorria, J., Warmling, D., Neves, C. M., Delziovo, C. R., \& Coelho, E. B. S. Prevalência e fatores associados da violência sexual contra a mulher: revisão sistemática (2017). Rev. salud pública, 19(6), 818-826.

Delziovo, C.R., Bolsoni, C. C., Nazário, N. O., Coelho, E. B. S. \& ELZIOVO, C. R. Características dos casos de violência sexual contra mulheres adolescentes e adultas notificados pelos serviços públicos de saúde em Santa Catarina, Brasil (2017). Cadernos de Saúde Pública, 33(6), 1-13.

Ercole, F. F., Melo, L. S., \& Alcoforado, C. L. G. C. Revisão integrativa versus revisão sistemática (2014). Revista Mineira de Enfermagem, 18(1), 9-12.

Garcia, A. L. C., \& Trajano, M. P. Violência sexual contra mulheres e saúde mental: um diálogo sobre norma técnica de prevenção e tratamento dos agravos resultantes da violência sexual contra mulheres e adolescentes (2018). CBSM, 10(25), 250-257.

Garcia, R. Neurobiologia do medo e fobias específicas. Learn Mem. 2017; 24, 462-471.

Juan C.S., Ana I A.R., Ana A.M \& Oscar C. O impacto da violência por parceiro íntimo nas atitudes sexuais, na assertividade sexual e no funcionamento sexual de homens e mulheres (2021). Int J Environ Res Saúde Pública, 18 (2), 594.

McQueen, K., Oikonen, J. M., Miller, A., \& Chambers, L. Sexual assault: womens voices on the health impacts of not being believed by police (2021). BMC, 21, 1-10.

Nelon, J. L., Pedro, K. T., Gilreath, T. D., Patterson, M.S., Holden, C., \& Esquivel, C. H. A latent class analysis of the co-occurrence of sexual violence, substance use, and mental heath in youth (2019). Substance Use \& Misuse, 54(12), 1938-1944.

Neta, R. A. D. B., Guimarães, S. O. P., Farias, M. C. C. \& Santos, L. A. Mulheres vítimas de abuso sexual em um município da Amazônia (2020). Ciência plural, 6(3), 123-136.

Oliveira, E. N., Jorge, B., \& Salete, M. (2007). Violência contra a mulher: sofrimento psíquico e adoecimento mental. Rev. Rene, 8(2), 93-100.

Oram, S., Khalifeh, H., \& Howard, L. Violência contra mulher e saúde mental (2017). Lancet Psychiatry, 4(2), 159-170.

Pedrosa, M., \& Zanello, V. (In)visibilidade da violência contra as mulheres na saúde mental (2016). Psicologia: Teoria e Pesquisa, 32(spe), 1-8.

Sharma, K. K., Vatsa, M., Kalaivani, M., \& Bhardwaj, D. Mental health effects of domestic violence against women in Delhi: A community-based study (2019). J Family Med Prim Care, 8(7), 2522-2527.

Sigurdardottir, S., \& Halldorsdottir, S. Sofrimento persistente: as graves consequências da violência sexual contra mulheres e meninas, sua busca pela cura interior e o significado do movimento \#MeToo (2021). Jornal internacional de pesquisa ambiental e saúde pública, 18 (4), 1849.

Taís, C.F., Lúcia H.M.F., Silva P.C.B., Carla C. Violência por parceiro íntimo contra mulheres: diagnóstico psicodinâmico operacionalizado (OPD-2) (2020). PLoS One, 15 (10), 1-18.

Trigueiro, T. H., Silva, M. H., Merighi, M. A. B. Oliveira, D. M., \& Jesus, M. C. P. O sofrimento psíquico no cotidiano de mulheres que vivenciaram a violência sexual: estudo fenomenológico (2017). Esc Anna Nery, 21(3), 1-7.

Zaina M., Gerry M., Diana A., Esther P., Donati M., Annapoorna D., Saidi K., Heidi S. “ Você não acha que é a violência me forçando a fazer sexo enquanto não estou feliz? "Conceituação feminina de sexo prazeroso e violência sexual por parceiro íntimo em Mwanza, Tanzânia (2020). Int J Environ Res Saúde Pública, 17 (21), 7937. 